\title{
Using computational fluid dynamics to study the effect of contact angle on microdroplet deformation
}

\author{
G. Rosengarten* $\quad$ D. Harvie ${ }^{\dagger} \quad$ J. Cooper-White
}

(received 27 October 2004, revised 21 March 2005)

\begin{abstract}
Computational fluid dynamics (CFD) is used to study the effect of contact angle on droplet shape as it moves through a contraction. A new non-dimensional number is proposed in order to predict situations where the deformed droplet will form a slug in the contraction and thus interact with the channel wall. We argue that droplet flow into a contraction is a useful method to ensure that a droplet will wet a channel surface without a trapped lubrication film. We demonstrate that when a droplet is larger than a contraction, capillary and
\end{abstract}

*School of Electrical and Computer Engineering, RMIT University, Australia. mailto: gary.rosengarten@rmit.edu. au

${ }^{\dagger}$ Dept. of Chemical and Biomolecular Engineering, University of Melbourne, Australia.

${ }^{\ddagger}$ Division of Chemical Engineering, University of Queensland, Australia.

See http://anziamj.austms.org.au/V46/CTAC2004/Rose for this article, (C) Austral. Mathematical Soc. 2005. Published April 28, 2005. ISSN 1446-8735 
Reynolds numbers, and fluid properties may not be sufficient to fully describe the droplet dynamics through a contraction. We show that with everything else constant droplet shape and breakup can be controlled simply by changing the wetting properties of the channel wall. CFD simulations with contact angles ranging from $30^{\circ}$ to $150^{\circ}$ shows that lower contact angles can induce droplet breakup while higher contact angles form contact-angle dependent shape slugs.

\section{Contents}

1 Introduction

C305

2 Numerical simulations

C307

2.1 Grid dependency and validation . . . . . . . . . .

3 Results

C310

3.1 Slug flow . . . . . . . . . . . . . . .

C310

3.2 Contact angle effects $\ldots \ldots \ldots \ldots \ldots$

C313

4 Conclusions

C317

References

C317

\section{Introduction}

The controlled deformation and breakup of droplets is important in a wide variety of systems including texture control processes for the food industry, or understanding cell flow through biological capillaries. Since the defining drop deformation work of Taylor in 1934 [17], there has been a considerable body of literature dealing with drop deformation in pure shear [7, e.g.] and 


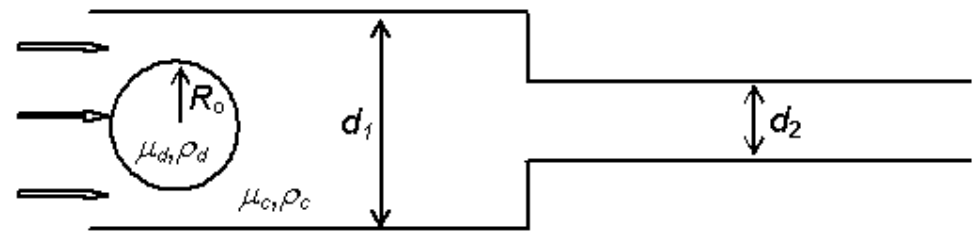

FIgURE 1: Schematic of contraction geometry.

planar extensional flows [8, e.g.]. There are fewer studies that deal with both simultaneously, which occurs in flow through a constriction [18, e.g.].

Extensional flows have been shown to be more energy efficient than shear flows in deforming drops [6] and unlike shear flows can deform and break up drops with any viscosity ratio. In simple shear flow a drop with a viscosity ratio $\lambda$ (the ratio of discrete phase viscosity to continuous phase viscosity), of 3.4 or more will not break up [8]. Flow through a contraction involves extensional fields in the contraction entry and exit regions, and shear only everywhere else. For the droplet deformation applications mentioned previously, useful droplet sizes will generally be less than approximately $200 \mu \mathrm{m}$. In order to manipulate and study droplets on this scale, microfluidic channels are extremely useful as they allow minimum quantities of the continuous phase, and the luxury of devising a channel layout with drop production and deformation components in a single channel system on a chip (a microfabricated capillary network for example). In microfluidics, velocities tend to be considerably lower than on macroscale systems meaning that interfacial tension forces often dominate over shear and inertial forces. The capillary number, the ratio of the viscous to interfacial tension forces, and the Reynolds number, the ratio of inertial to viscous forces, are (see Figure 1)

$$
\mathrm{Ca}=\frac{\mu_{c} v_{1}}{\sigma} \quad \text { and } \quad \operatorname{Re}_{d_{1}}=\frac{\rho_{c} v_{1} d_{1}}{\mu_{c}},
$$

where $\mu_{c}$ is the continuous phase viscosity, $v_{1}$ is mean upstream velocity, $\sigma$ is the interfacial tension, $\rho_{c}$ is the continuous phase density, and $d_{1}$ is the 
upstream channel diameter. In most applications droplet viscosities will be considerably larger than the continuous phase viscosity so the best way to deform and break droplets is in extensional flow, which is easily achieved by varying the microchannel dimension to form a constriction or a contraction as shown in Figure 1.

Recently it has been shown that two-phase flow patterns in microchannels depend on wetting properties of the fluids relative to the channel walls [5]. This is not surprising given the high surface to volume ratios found in microchannels, which allow surface properties to affect the bulk flow. Here we propose the use of hydrodynamic forces and surface forces to deform and possibly break droplets in microchannels. To that end we use computational fluid dynamics (CFD) with the volume of fluid method (VOF) to simulate the effect that the surface free energy, or its macroscale manifest, the contact angle, has on the way a droplet is deformed as it passes through a microcontraction. Important issues that are addressed are: the determination of conditions that allow surface interaction through the contraction; and the presence of a thin lubricating film shielding the droplet or slug from surface effects.

\section{Numerical simulations}

In order to predict the dynamics of two immiscible fluids, including surface tension and contact angle effects, we use the volume of fluid (VOF) code contained in commercial package CFD-ACE [http:www.cfdrc.com], which is based on the VOF method first proposed by Hirt and Nichols in 1981 [10], and recently extended by Rider et al. [16]. The VOF method involves tracking the volume fraction of each fluid in the computational cells via solving the transport equation for the volume fraction of one species, $F$ :

$$
\frac{\partial F}{\partial t}+\nabla \cdot(F \boldsymbol{v})=0
$$


An upwind scheme with the Piecewise Linear Interface Construction (PLIC) method [13] calculates the interface shape from the values of $F$ in each cell. In this scheme each cell has a unique surface normal that is used to compute the surface curvature from cell to cell. Interfacial tension is included as a source term in the momentum equation.

The contact angle is implemented in the code not as a boundary condition, but within the surface tension model in the solver. The fluid contact angle at the wall is used to rotate the surface normal in the cells adjacent to the wall and thus to adjust the curvature and interface shape near the wall. This method was first described by Brackbill et al. in 1992 [3] and is implemented using $\hat{n}=\hat{n}_{\text {wall }} \cos \theta+\hat{n}_{t} \sin \theta$, where $\hat{n}$ is the unit surface normal, and $\hat{n}_{\text {wall }}$ and $\hat{n}_{t}$ are the unit vectors normal and tangential to the wall. The rotated surface normal is then used to adjust the curvature of the surface, $\kappa$, near the wall using $\kappa=\nabla \cdot \boldsymbol{n}$.

This is a macroscopic forcing of the effects due to nanoscale interactions between the two fluids and the surface, yet it generally mimics observed phenomena and has been used successfully for some time [14, and references therein]. We use a static contact angle dynamic contact angles are not taken into account.

No-slip boundary conditions at the walls are imposed. However, this does not mean that the contact line with the wall cannot move, as the code integrates over the whole cell adjacent to the wall to compute the mass flux of the second phase, which, when using a finite grid size, will average out to give an apparent finite interface velocity at the wall. The numerically induced slip is also described by Renardy et al. [15] who showed that the contact line motion is not very sensitive $(<5 \%)$ to the addition of artificial slip lengths with magnitudes in the order of the grid size. All droplet shapes are shown as contours of $F=0.5$. 
a

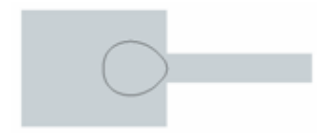

\section{i}
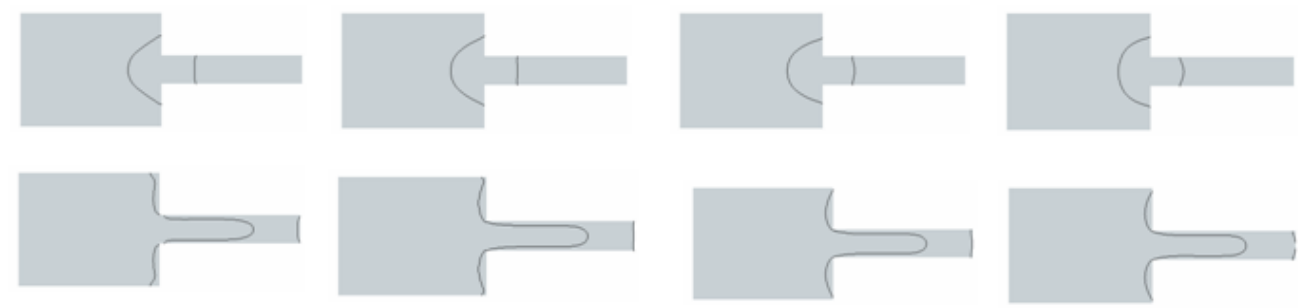

ii
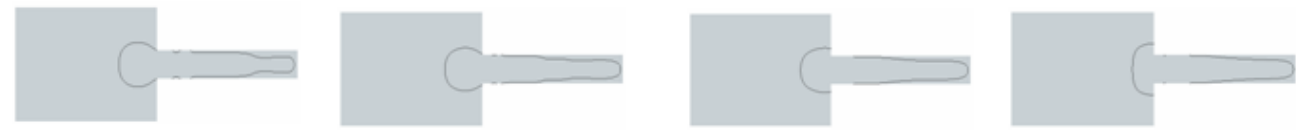

FiguRE 2: Grid dependence of drop deformation through a 4:1 contraction, $\mathrm{Ca}=5 \times 10^{-3}, \mathrm{Re}_{d_{1}}=10$ and contact angle $90^{\circ}$, (i) for a $2 \mathrm{~d}$ geometry at 3 different times and (ii) an axisymmetric geometry. a) 4 cells across contraction radius, b) 8 cells, c) 16 cells d) 32 cells.

\subsection{Grid dependency and validation}

It is not expected that a VOF code will be truly grid independent when simulating moving contact lines along a surface as the grid size near the wall will affect how the contact line moves [15]. In fact, the actual physics of moving contact lines itself is not well resolved [1], and other simulation methods generally need to use some kind of arbitrary slip or precursor films even for full wetting systems [4]. This ensures that an infinite force is not needed to drive the fluid at the 3-phase interface. Our aim is to ensure that there is not a significant variation in results as the grid resolution is changed. We examined the grid dependency of the solution for a case with a moving contact line by increasing the grid resolution by a factor of 8 , 
from 4 to 32 cells in the contraction radius. Square cells were used so the values correspond to to 800 to 51200 cells for both a 2D rectangular and an axisymmetric geometry. The effect of the shape of a droplet as it moves through a contraction is shown in Figure 2, where small differences are seen in the shape of the deforming droplet as a function of grid density. Three time steps are shown for the $2 \mathrm{D}$ case and one single time for the axisymmetric case. Clearly, the deformation has not reached a grid independency, but the major features do not change considerably between cases $\mathrm{c}$ and $\mathrm{d}$. Thus the rest of the simulations use grid c.

We have also compared our free stream droplet shape results with previous studies. Figure 3 shows a steady state droplet shape with velocity vectors relative to the droplet velocity. Both the shape and velocity fields compare very well, giving us confidence in the CFD-ACE code.

\section{Results}

\subsection{Slug flow}

When a droplet that is initially larger than a contraction moves through the contraction, there are essentially two scenarios that can occur. The droplet can either form a slug in the contraction or glide through without touching the walls (the two scenarios are shown in Figure 4). Although the two scenarios may not always be exactly delineated, slug-like flow is required for surface force control of droplet deformation. For low upstream Reynolds numbers $(\mathrm{Re} \leq 1)$ the deformation of a droplet travelling though a contraction is due to a balance of the viscous extensional force and interfacial tension forces. The viscous extensional force tends to stretch the drop out in order to follow the stream lines while the interfacial tension force tends to keep the drop in a spherical shape. The balance of the two forces is generally gauged by the capillary number, which is given in equation (1) but may also 

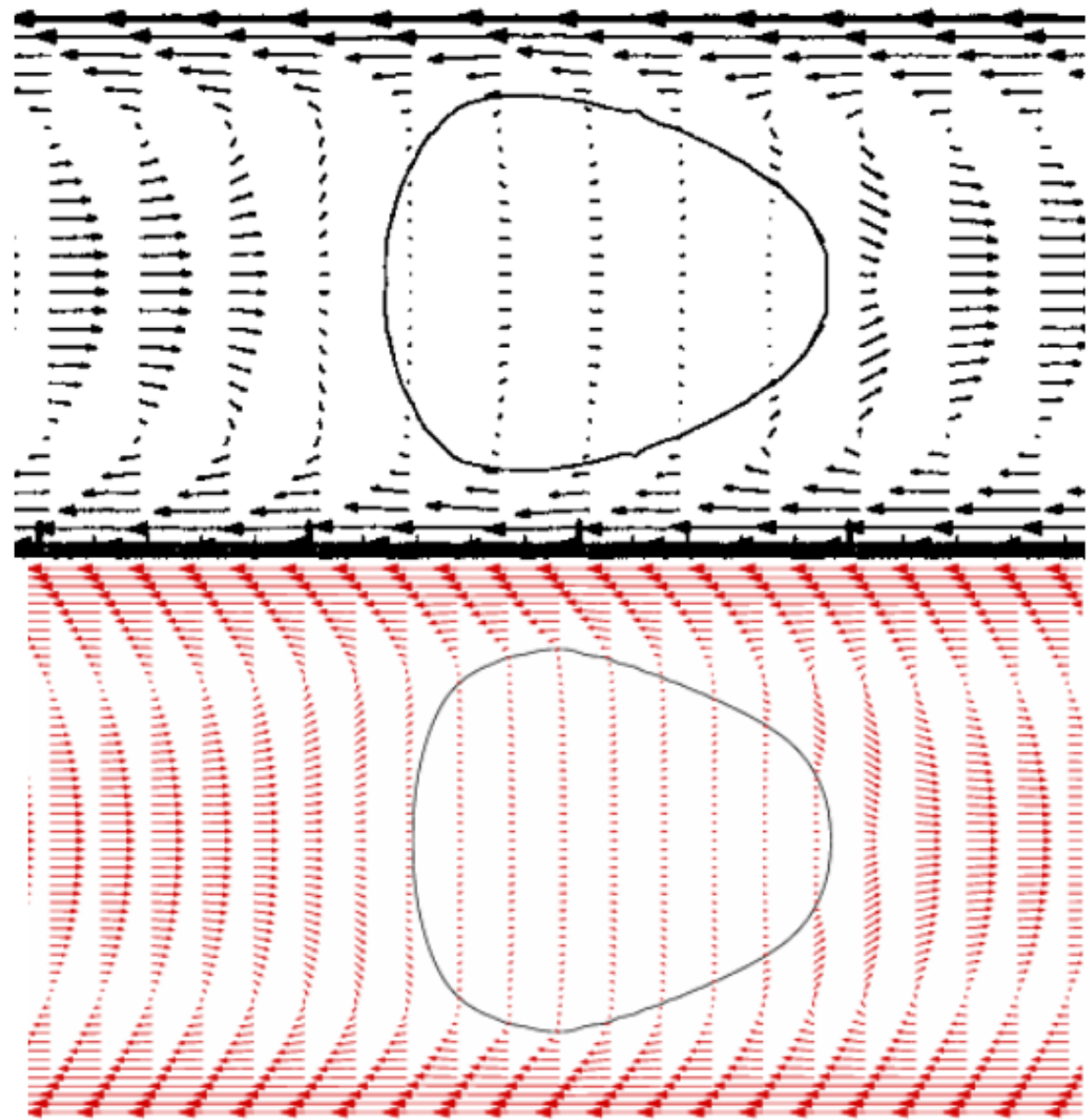

FiguRE 3: Steady-state droplet shape and velocity field relative to droplet velocity for a neutrally buoyant drop in axisymmetric Poisuille flow, $\mathrm{Ca}=$ 0.1, $\lambda=10$. (a) From [12] (b) Current simulations using CFD-ACE. 


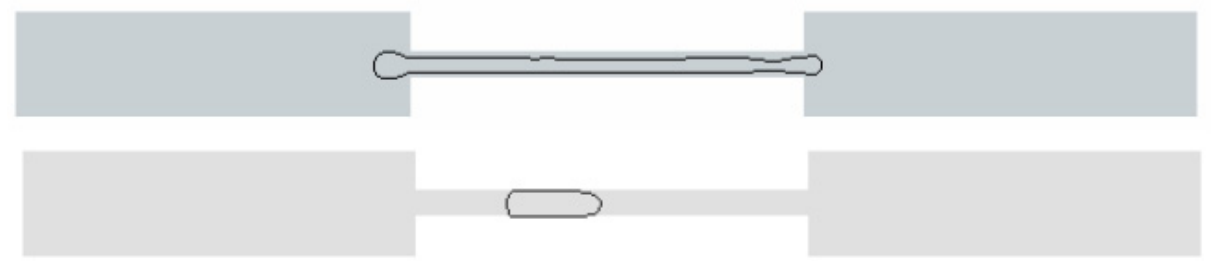

Figure 4: Example of a filament and a slug flow through a capillary, for non-wetting walls. The filament conditions are $\mathrm{Re}=1, \mathrm{Ca}=0.05$ and $\lambda=0.1$. The slug conditions are $\mathrm{Re}=1, \mathrm{Ca}=0.005$ and $\lambda=10$.

be written as

$$
\mathrm{Ca}=\frac{\mu_{c} \dot{\gamma} R_{0}}{\sigma},
$$

where $\dot{\gamma}$ is the extensional strain rate, and $R_{0}$ is the drop diameter (see Figure 1)

The average extensional strain rate due to a contraction can be approximated by $\left(v_{2}-v_{1}\right) / L$, where $v_{2}-v_{1}$ is the difference in mean velocity between the two upstream and downstream channels and $L$ is the average distance over which this change takes place. It has been established experimentally that for $\operatorname{Re} \leq 1$ and for contraction ratio $\beta \geq 4$ (where $\beta=d_{1} / d_{2}$ ), that the entry lengths and upstream vortex attachment length for flow through a contraction can be approximated by $0.25 d_{2}$ and $0.17 d_{1}$ respectively [2]. If we assume the sum of these two lengths approximates the extensional deformation regime through a contraction, then the extensional strain rate

$$
\dot{\gamma}=\frac{v_{2}-v_{1}}{0.25 d_{2}+0.17 d_{1}} .
$$

For an axisymmetric contraction $v_{2}=\beta^{2} v_{1}$, therefore

$$
\dot{\gamma}=\frac{v_{1}\left(\beta^{2}-1\right)}{d_{1}(0.25 / \beta+0.17)} .
$$


Substituting equation (5) into equation (3) gives the contraction capillary number

$$
\mathrm{Ca}_{\text {con }}=\frac{\mu_{c} v_{1}}{\sigma} \frac{R_{0}\left(\beta^{2}-1\right)}{d_{1}(0.25 / \beta+0.17)}=\mathrm{Ca} \Omega,
$$

where $\Omega$ is the contraction factor.

At Reynolds numbers greater than approximately 1 but less than approximately 100 inertia becomes important but not dominant, and surface tension then competes with inertia and with the extensional force. By a simple order of magnitude analysis of the momentum equation, a new number can be defined that is the ratio of surface tension to the sum of inertia and extensional viscous forces:

$$
\mathrm{S}_{\text {con }}=\frac{1}{\mathrm{Ca}_{\mathrm{con}}(1+\mathrm{Re})} .
$$

A number of simulations have been performed, and the droplet shapes in the contraction categorised into either slugs or filaments. In general a slug was defined when the film between the slug and the wall was less than about $1 / 30$ th of the contraction diameter (one cell wide). The results are summarised in Figure 5 which contains 4:1 and 6:1 contractions and viscosity ratios of $0.1,1$ and 10 . Using the contraction capillary number the results collapse to the two delineated regions. Clearly, $\mathrm{S}_{\mathrm{con}}$, equation (7), is a good indicator of the boundary between the two regions.

\subsection{Contact angle effects}

For the surface properties to affect the droplet shape, the droplet either has to wet the surface or be close enough to the surface for long range van der Waals forces to have an effect $(<100 \mathrm{~nm}$ which is smaller than the grid resolution). Therefore, a droplet that is initially completely detached from the wall must approach the wall and drain the continuous fluid away. A sharp contraction is ideal for this as the droplet essentially approaches a point (or 


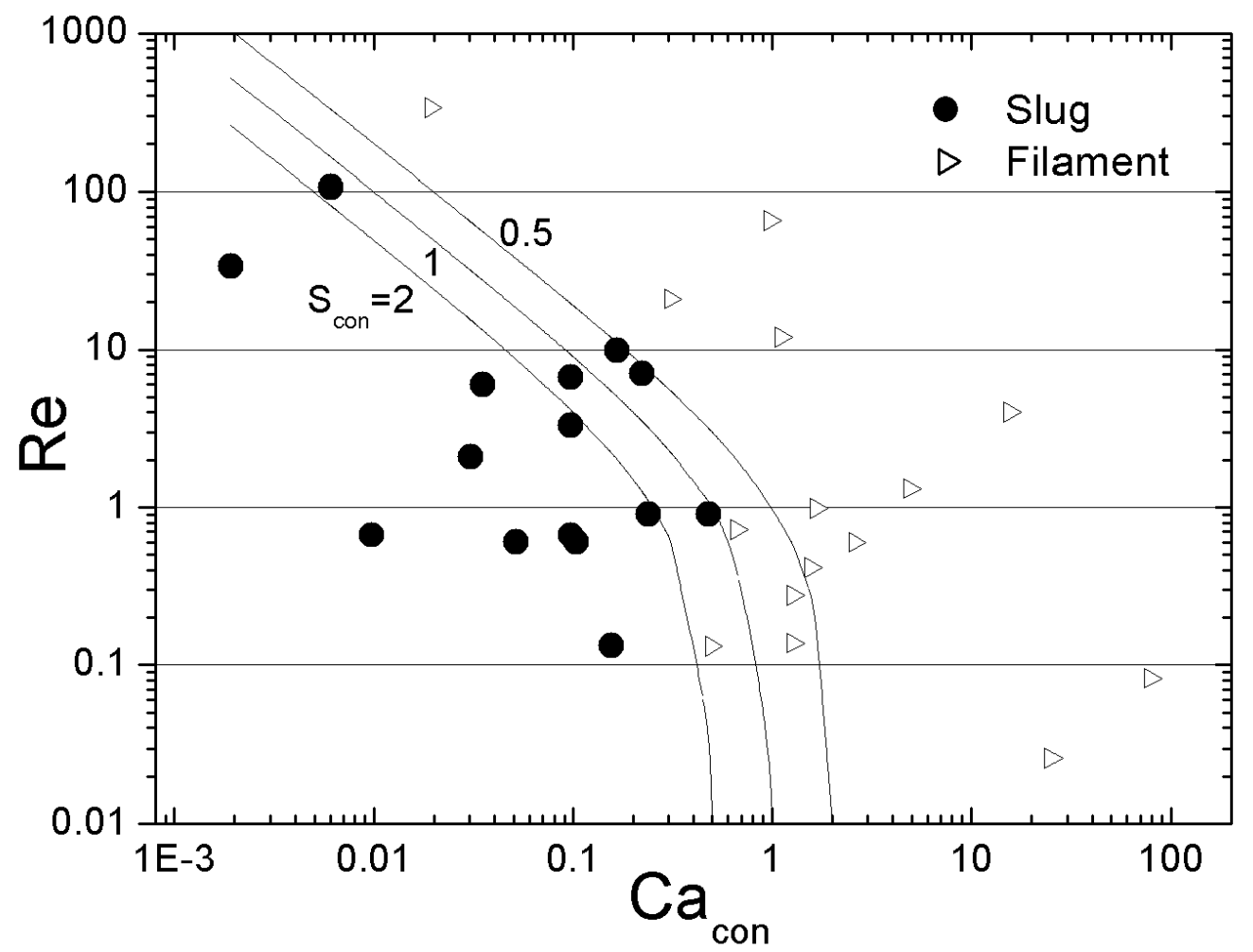

FiguRE 5: Reynolds number versus contraction capillary for flow through a 4:1 contraction and a 6:1 contraction under a range of conditions. Demarcation between slug and filament flow is correlated to $\mathrm{S}_{\text {con }}$. 
Contact Angle $30^{\circ}$
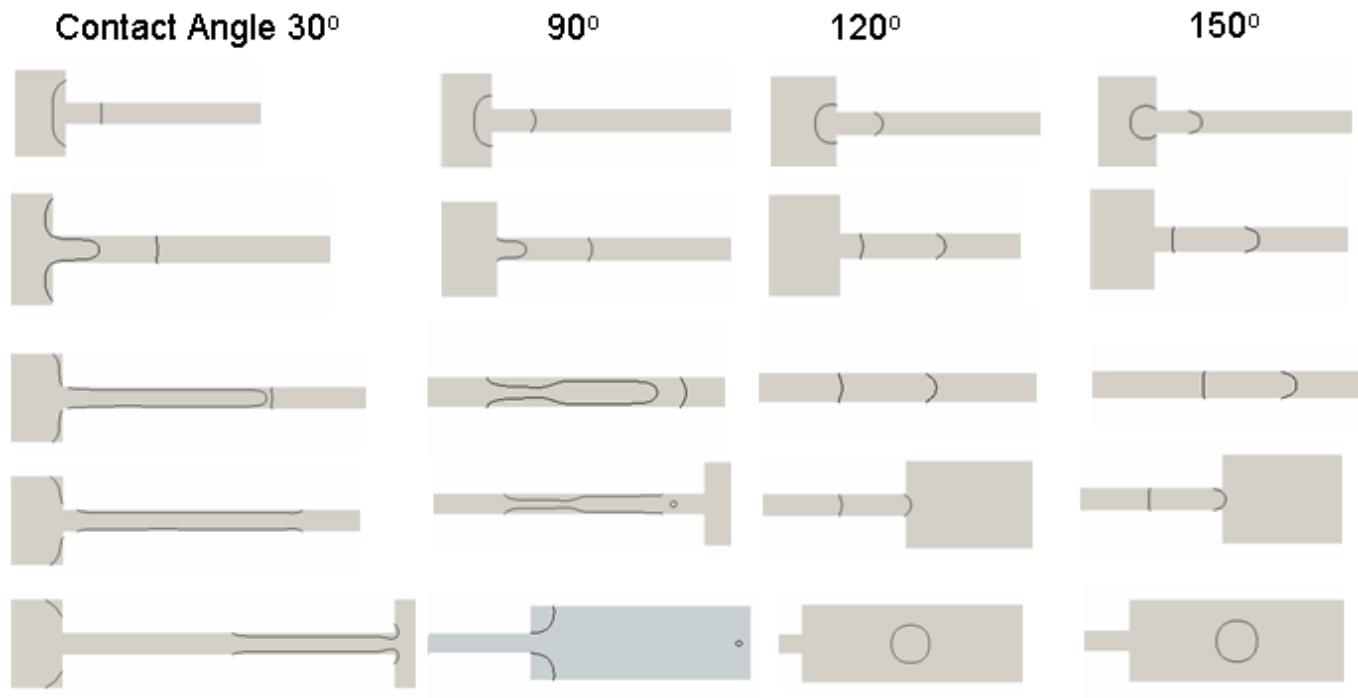

Figure 6: Contact angle effects in a rectangular 4:1 channel for $\mathrm{Ca}=5 \times$ $10^{-3}, \mathrm{Ca}_{\mathrm{con}}=0.16$ and $\lambda=10$.

in reality a very small surface) meaning that minimal amounts of film needs to be drained. Thus film drainage times will not be a limiting factor. Similar contact angle effects have been shown using Lattice Boltzmann simulations of a collapsing confined fluid thread [9].

Many studies have shown that a moving slug will have a thin film, between it and the channel wall, which scales approximately as $\mathrm{Ca}^{2 / 3}[11$, and references therein]. However, for slugs that are initially wetting we have observed experimentally and numerically a critical capillary number for the drop to detach from the surface and form the classical film. This is very similar to liquid drop detachment from a solid surface due to shear, which occurs after exceeding critical shear rates [1] that are of the same order as observed in our microchannels.

Given that a contraction forms ideal flow conditions for a droplet to wet 
Contact Angle $30^{\circ}$

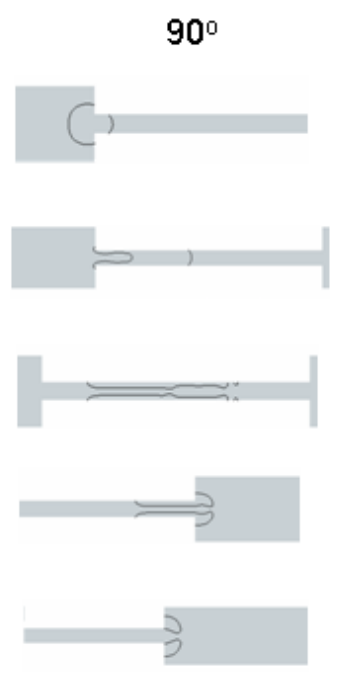

$120^{\circ}$

$150^{\circ}$
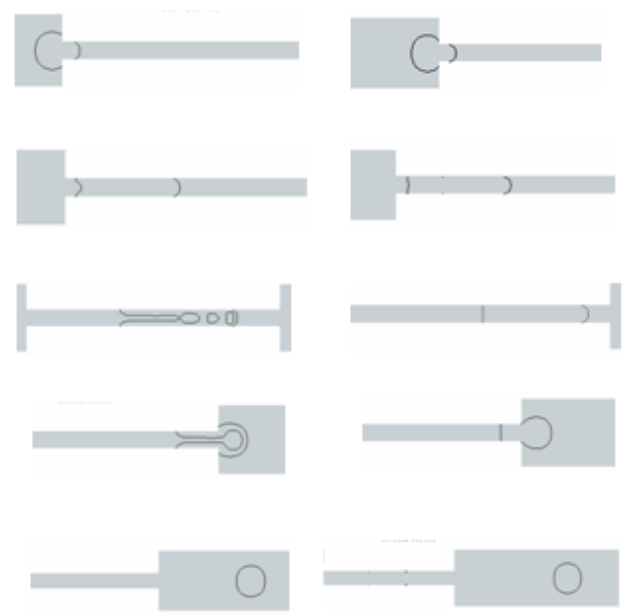

Figure 7: Contact angle in a axisymmetric 4:1 channel for $\mathrm{Ca}=4.3 \times 10^{-4}$, $\mathrm{Ca}_{\text {con }}=1.4 \times 10^{-2}$ and $\lambda=5$.

a surface, then the contact angle that the drop makes with the surface will have a large effect on what happens to the droplet. This is clearly indicated in Figures 6 and 7 that show the effect of changing the contact angle on droplet deformation through two-dimensional rectangular and axisymmetric channels respectively. For a contact angle of $30^{\circ}$ the droplet wants to wet the contraction, and some of the dispersed phase gets trapped in the upstream corners, while the rest of the droplet forms annular flow. As the contact angle increases, the forces holding the droplet to the wall decrease and the droplet proceeds into the contraction. In the $90^{\circ}$ case the slug formed in the contraction rapidly deforms due to partial wall adhesion and the parabolic velocity profile. This results in breakup and, as the residual slug exits the contraction, some is trapped in a exit corner recirculation zone. For the $120^{\circ}$ and $150^{\circ}$ cases the slug front and back curvatures vary and change the shape of the slug. Similar deformation occurs in the axisymmetric case except that breakup occurs at higher contact angles due to higher (by a factor of 4) 
extension rates.

\section{Conclusions}

We have shown that under certain conditions surface forces affect the shape of a droplet passing through a contraction. In order to help predict those conditions, we introduced a new contraction capillary number and an associated contraction surface tension number that is the ratio of the surface tension force to the sum of inertia and extensional viscous forces. Under conditions where surface forces affect the droplet phase, the capillary number, Reynolds number and fluid properties are not sufficient to uniquely describe the motion of droplets through contractions. We have shown that the droplet contact angle can have a large effect on the droplet dynamics and thus should be taken into account and possibly utilised when designing microfluidic droplet manipulation systems.

\section{References}

[1] S. Basu, K. Nandakumar, and J. Masliyah. A model for detachment of a partially wetting drop from a solid surface by shear flow. Journal of Colloid and Interface Science, 190:253-257, 1997. http://www. ingentaconnect. com/content/ap/cs/1997/00000190/ 00000001/art04856 C309, C315

[2] D. Boger. Viscoelastic flow through contractions. Ann. Rev. Fluid Mech., 19:157-182, 1987.

http://dx.doi.org/10.1146/annurev.fl.19.010187.001105 C312

[3] J. U. Brackbill, D. Kothe, and C. Zemach. A continuum method for modeling of surface tension,. J. Comp. Phys., 100:335-354, 1992. C308 
[4] J. Diez, L. Kondic, and A. Bertozzi. Global models for moving contact lines. Phyiscal Review E, 63:011208-1-13, 2001. C309

[5] R. Dreyfus, P. Tabeling, and H. Willaime. Ordered and disordered patterns in two-phase flows in microchannels. Physical Review Letters, 90(14):144505-1, 2003. C307

[6] L. Erwin. Principles of laminar fluid/fluid mixing. In Chris Rauwendaal, editor, Mixing in Polymer Processing. Marcel Dekker, 1991. C306

[7] S. Guido, M. Simeione, and F. Greco. Deformation of a newtonian drop in a viscoelastic matrix under steady shear flow. experimental validation of slow flow theory. J. Non-Newtonian Fluid Mech, 114:65-82, 2003. C305

[8] J. W. Ha and L. G. Leal. An experimental study of drop deformation and extensional flow at high capillary number. Physics of fluids, 13(6):1568-1576, 2001. http://dx.doi.org/10.1063/1.1358306 C306

[9] J. Hagedorn, N. Martys, and J. Doughlas. Breakup of a fluid thread in a confined geometry: droplet-plug transition, perturbation sensitivity, and kinetic stabilzation with confinement. Physical Review E, 69:056312-1-12, 2004. C315

[10] C. W. Hirt and B. D. Nichols. Volume of fluid (vof) method for the dynamics of free boundaries. Journal of Computational Physics, 39:201-225, 1981. C307

[11] S. R. Hodges, O. E. Jensen, and J. M. Rallison. The motion of a viscous drop through a cylindrical tube. J. Fluid Mech., 501:279-301, 2004. http://dx.doi.org/10.1017/S0022112003007213 C315

[12] C. Kaliakatsos and S. Tsangaris. Motion of deformable drops in pipes and channels using navier-stokes equations. Int. J. Numer. Meth. 
Fluids, 34:609-626, 2000. http:

//www3.interscience.wiley.com/cgi-bin/abstract/75504241 C311

[13] D. B. Kothe, W.J. Rider, J. Mosso, and J. S. Brock. Volume tracking of interfaces having surface tension in two and three dimensions. AIAA, pages 96-0859, 1996. C308

[14] M. Pasandideh-Fard, Y. M. Qiao, S. Chandra, and J. Mostaghimi. Capillary effects during droplet impact on a solid surface. Physics of fluids, 8(3):650-659, 1996. http://dx.doi.org/10.1063/1.868850 C308

[15] M. Renardy, Y. Renardy, and J. Li. Numerical simulation of moving contact line problems using a volume-of-fluid method. J. Comp. Phys., 171:343-263, 2001. C308, C309

[16] W. J. Rider, D. B. Kothe, S. J. Mosso, and J. I. CerruttiHochstein. Accurate solution algorithms for incompressible multiphase fluid flows. AIAA, pages 95-0699, 1995. C307

[17] G. I. Taylor. The formation of emulsions in definable fields of flow. Proc. Roy. Soc., 146A:501-523, 1934. C305

[18] T. M. Tsai and M. J. Miksis. Dynamics of a drop in a constricted tube. J. Fluid Mechanics, 274:197-217, 1994. C306 